\title{
Iris segmentation: state of the art and innovative methods
}

\author{
Ruggero Donida Labati, Angelo Genovese, Vincenzo Piuri, and Fabio Scotti
}

\begin{abstract}
Iris recognition is nowadays considered as one of the most accurate biometric recognition techniques. However, the overall performances of such systems can be reduced in non-ideal conditions, such as unconstrained, on-the-move, or noncollaborative setups.

In particular, a critical step of the recognition process is the segmentation of the iris pattern in the input face/eye image. This process has to deal with the fact that the iris region of the eye is a relatively small area, wet and constantly in motion due to involuntary eye movements. Moreover, eyelids, eyelashes and reflections are occlusions of the iris pattern that can cause errors in the segmentation process. As a result, an incorrect segmentation can produce erroneous biometric recognitions and seriously reduce the final accuracy of the system.

This chapter reviews current state-of-the-art iris segmentation methods in different applicative scenarios. Boundary estimation methods will be discussed, along with methods designed to remove reflections and occlusions, such as eyelids and eyelashes. In the last section, the results of the main described methods applied to public image datasets are reviewed and commented.
\end{abstract}

\section{Introduction}

The number of deployed biometric systems is continuously growing all over the world in security-oriented and everyday applications [61]. In 2011, the related market reached the amount of 5 billion dollars with a significant positive trend, and is expected to reach 12 billion dollars by the end of 2015 [1].

Ruggero Donida Labati, Angelo Genovese, Vincenzo Piuri, and Fabio Scotti Università degli Studi di Milano, Department of Information Technology, I-26013 Crema (CR), Italy. e-mail: \{ruggero.donida, angelo.genovese, vincenzo.piuri, fabio. scotti\}@unimi.it 
(a)

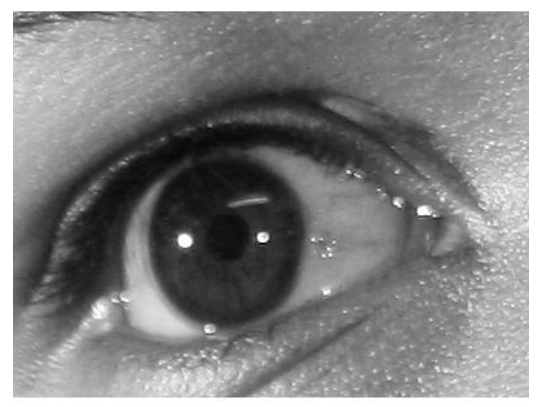

(b)

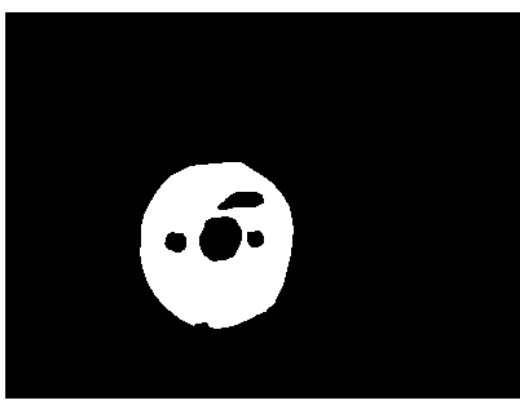

Fig. 1 Segmentation of the iris pattern. Starting from an eye image (a), this process estimates a binary mask (b) in order to classify the image pixels belonging to the iris pattern. The binary mask excludes occlusions (such as eyelids, eyelashes) and reflections. Additional information can also be extracted, such as the pupil/iris centers and radii, or a secondary binary mask containing the map of the occlusions superimposed on the iris pattern

Events such as terrorism acts or computer hacking have led to a greater interest and, consequently, to major developments in the safety and protection of data, goods and individuals. In this context, iris recognition systems offer a fast and secure method for the recognition of individuals, with the ability to perform accurate identification even with databases composed by several millions of enrolled templates [18]. Examples of applications include the passenger identification in major city airports or border controls, where accuracy and speed are of uttermost importance $[38,44,20,21]$. Moreover, biometric technologies based on the iris recognition are becoming more and more affordable and widespread due to the integration of iris scanners in mobile phones [13, 12] and embedded systems [45].

One of the major issues in the deployment of iris recognition systems is the performance decreasing of such systems in non-ideal contexts, such as in unconstrained, on-the-move, covert, or non-collaborative setups.

In particular, a critical step of the biometric recognition process is related to the ability of the image processing system to effectively locate and separate the iris pattern in the input face/eye image. This process, referred as iris segmentation (Fig. 1), still presents many challenges. The iris region, in fact, is a small moving area, and often occluded by eyelids and eyelashes. These problems are especially relevant when the user does not face the camera or is walking from a considerable distance. Moreover, the correct detection of the iris boundaries and the removal of the occlusions are directly related to the accuracy of the iris recognition system.

Novel approaches and currently research trends in the detection of the iris region aim to achieve a more robust and accurate localization of the iris pattern even in non-ideal conditions, in order to allow the application of iris recognition systems in a broader range of scenarios. Similarly, the ability to correctly segment and enhance the iris images captured even from a great distance could result in an even higher control over critical transit points. 


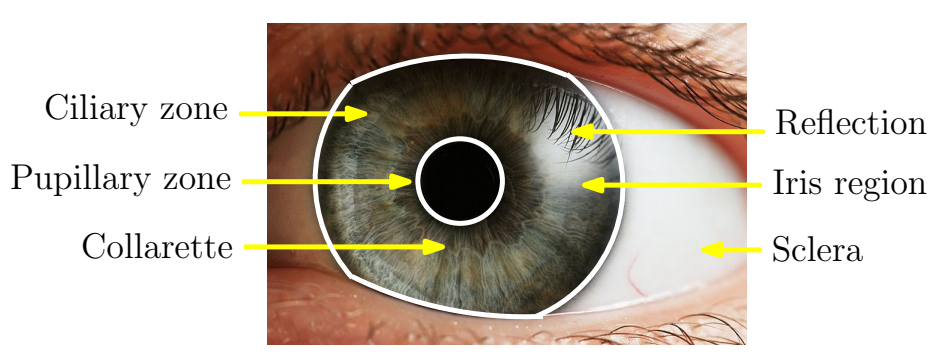

Fig. 2 Example of an iris pattern captured in visible light and its constituting elements

This chapter is focused on the iris segmentation and reviews the main and novel approaches in this field. After a short overview of an iris recognition system, the problem of iris segmentation is presented. The most used methods for the iris segmentation, which estimate the iris boundaries as two circumferences, are reviewed. Then, a description of the methods based on other a-priori approximation models is presented. Novel approaches are subsequently divided in methods based on the analysis of local characteristics, methods based on active contours, and methods that perform a hybrid or incremental segmentation. Some methods, which do not fall in any specific category, are also described, followed by a survey of the methods for the detection of occlusions and reflections. Last of all, a discussion of the most important public datasets, evaluation metrics, and recent performances of iris segmentation methods is proposed.

\section{Segmentation of the iris pattern}

The human iris is an annular-shaped area between the pupil and the sclera (Fig. 2), which is stable from the eighth month of gestation [17]. A normal iris pattern shows hundreds of random texture features in its pigmentation. These features are unique for each individual, also in the case of homozygote twins. Moreover, the iris is well protected by the cornea and it is not affected by external factors, differently, for example, from fingerprints, which are sensitive to skin conditions [44].

The high degree of randomness of the iris pattern is the key feature exploited in the iris biometric systems to produce reliable and robust biometric templates that allow to obtain a very high accuracy of the recognition process.

The schema of a biometric system based on the iris trait is depicted in Fig. 3. The four modules that compose an iris recognition system are: image acquisition, image segmentation, analysis and representation of the iris features, matching of iris representations [7].

The image acquisition module is usually composed by cameras that capture images in the near-infrared range $(700-900 \mathrm{~nm})$. The ISO Iris Image Standard requires that the length of the iris diameter is at least 200 pixels [2]. The user cooperation is usually required in order to properly capture the iris image. 


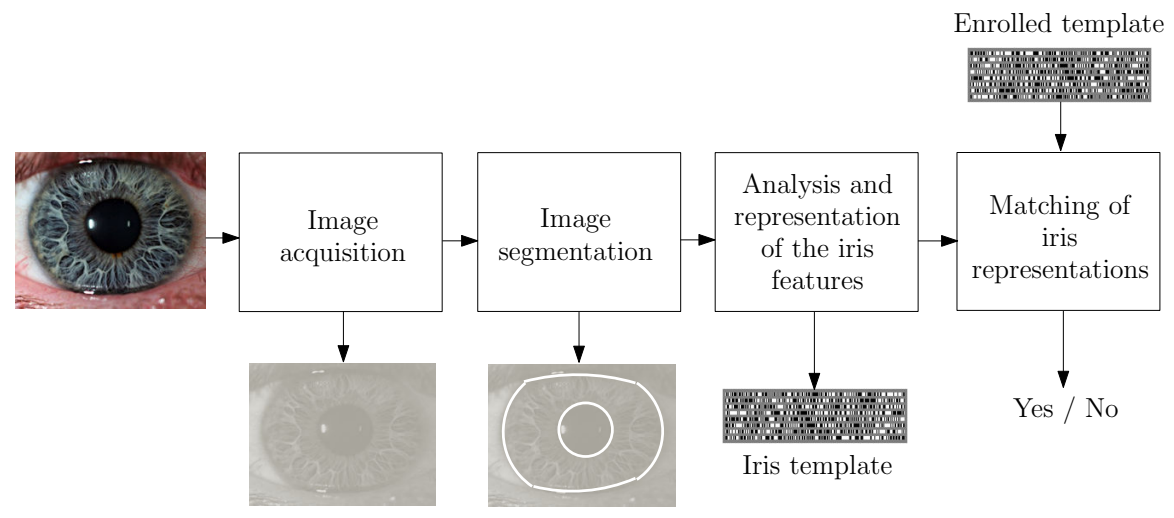

Fig. 3 Schema of an iris recognition system

The image segmentation module performs the localization of the iris in the image and removes the areas corresponding to eyelids, eyelashes, reflections, and shadows [34]. Typically, the iris segmentation is the most time-consuming step [17] and its effectiveness is relevant because the obtained accuracy strongly influences the results of the biometric system [47]. An incorrectly segmented iris, in fact, can result in errors of the matching module.

The next module is based on algorithms that analyze the segmented iris image and extract the distinctive features from the iris pattern. These features are then used in order to compute an abstract representation, called template. One of the most commonly used templates is the Iriscode [17].

The last module computes a matching distance between two or more templates, in order to determine if they belong the same person. In most of the cases, the matching value is computed as the Hamming distance between shifted templates $[17,44]$.

Since the iris region of the eye is a relatively small area, wet, and constantly moving due to involuntary eye movements, a robust segmentation is needed in order to correctly identify the region of the captured image that should be considered during the subsequent steps of the biometric recognition process. The iris segmentation is usually performed in two steps: first the inner and outer boundaries of the iris region are estimated, then occlusions and reflections are detected and removed.

Problems related to reflections and occlusions are particularly relevant. In many situations, in fact, the iris pattern is not completely visible because the eyelids cover a portion of the region of interest (Fig. 4a). Eyelashes are often present in the input image and can occlude big regions the iris (Fig. 4b). Moreover, since the eye is a wet convex surface, reflections can occur due to the presence of environmental light sources (Fig. 4c). Other important problems are related to the size variability of the same iris in different images, and to off-axis gaze situations.

The described problems have a higher relevance for biometric systems that are based on iris images captured by less-constrained acquisition setups (e.g. in visible light conditions, at a great distance, on the move). A qualitative comparison between 
(a)

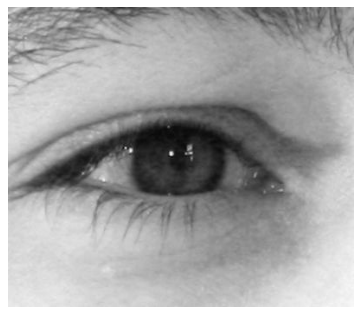

(b)

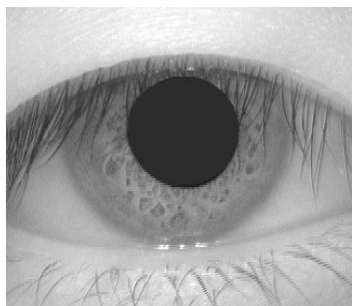

(c)

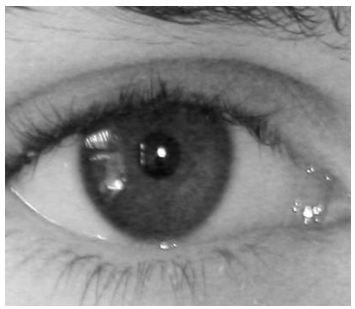

Fig. 4 Examples of reflections and occlusions: (a) eyelids; (b) eyelashes; (c) reflections

(a)

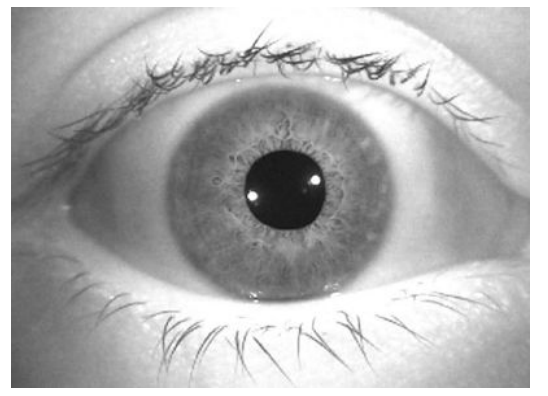

(b)

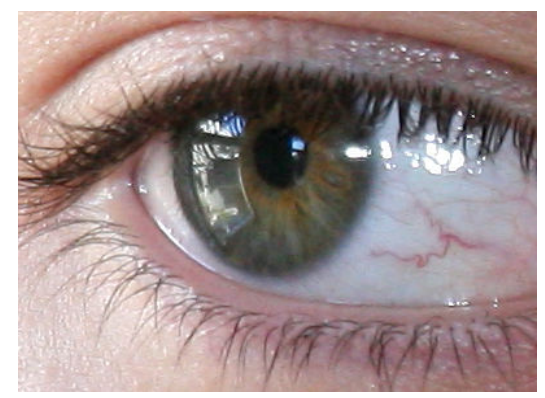

Fig. 5 Comparison between iris images captured in constrained and unconstrained conditions: (a) an image captured in constrained conditions and infrared light; (a) an image captured in unconstrained conditions and natural light. The image (b) presents problems related to reflections, occlusions, and off-axis gaze

an iris image captured in constrained conditions by using infrared light lamps and an image captured in unconstrained conditions is shown in Fig. 5.

The result of the iris segmentation step consists in a binary image describing the region of interest. Fig. 1 shows an example of a close-up eye image and the corresponding binary segmented image.

Most of the iris recognition methods use the binary image obtained by the segmentation step in order to define the area of the image that should be considered in the matching of iris representations. However, the matching step of many iris recognition systems requires supplementary information, such as the parameters describing a geometrical shape that approximates the iris area. In most of the cases, these parameters are the radius and the center coordinates of the circumferences that approximate the inner and outer iris boundaries.

In the literature, the majority of the iris boundary detection methods approximates the iris by two concentric circumferences. These methods are based on integro-differential operators $[17,16,70,60,65]$, the Hough transform $[79,27,55$, $75,73,48]$, iterative algorithms [10, 22, 71], or different techniques [5, 58, 42, 37]. Other methods use more complex a-priori models (such as ellipses) [83, 53, 64, 76, 


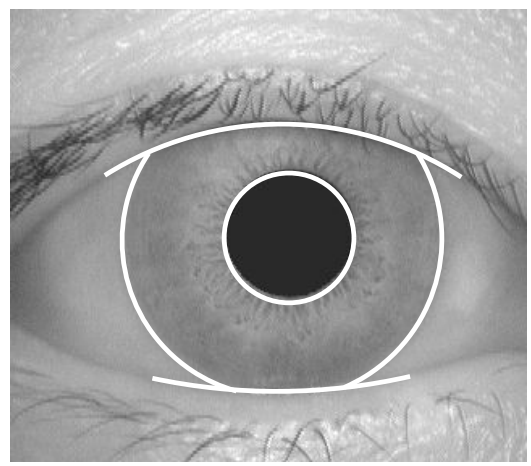

Fig. 6 Example of a result obtained by the methods that approximate the iris boundaries by two circumferences and the eyelid boundaries as parabolic arcs

29] in order to approximate the iris boundaries. There are also methods based on the analysis of local image features [53, 43, 41, 26, 9, 62, 59]. Methods based on active contours techniques are described in $[69,63,82,39]$, and some hybrid and incremental approaches are presented in $[19,25,24,23,34,83]$. There are also iris segmentation approaches that are difficult to classify, for example the methods proposed in [14, 46, 74].

Some methods specifically deal with the problem of removing occlusions, such as eyelids, eyelashes, and shadows. Some of the proposed algorithms are described in $[83,65,63,26,25,33,34,74,80,81,3,40]$.

\section{Methods that approximate the iris boundaries by two circumferences}

The estimation of the iris boundaries by means of circumferences is one of the most applied methods in the literature. In particular, the inner and outer iris boundaries of the iris are approximated by two circumferences and, similarly, the upper and lower eyelid boundaries are then approximated by parabolic arcs. Fig. 6 shows a result obtained by one of these methods applied on an iris image captured under IR illumination.

This technique was first proposed in $[17,16]$ and is one of the most used segmentation methods in the literature. The first step consists in the estimation of two circumferences that approximate the inner and outer iris boundaries. Then, the upper and lower eyelid boundaries are searched and approximated by two curves. The estimation of the circumferences that describe the inner and outer boundaries of the iris is performed by an algorithm that iteratively tests different possible center coordinates and radius values. The result of this algorithm is the circumference corresponding to the greater value resulting from the application of an integro-differential operator. The used integro-differential operator is defined as: 


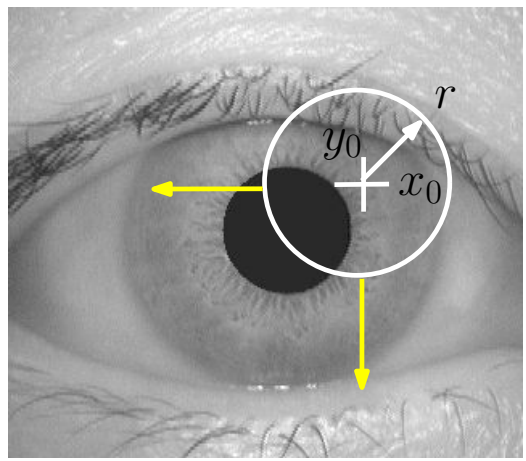

Fig. 7 Behavior of the iris segmentation method based on the integro-differential operator proposed in [17]

$$
\max _{\left(r, x_{0}, y_{0}\right)}\left|G_{\sigma}(r) * \frac{\alpha}{\alpha_{r}} \oint_{r, x_{0}, y_{0}} \frac{I(x, y)}{2 \pi r} d s\right|
$$

where $I(x, y)$ is the image containing the iris, $r$ is the evaluated radius, $\left(x_{0}, y_{0}\right)$ are the candidate coordinates of the center, and $d s$ represents the circular arc. The term $G_{\sigma}(r)$ is a Gaussian mask with scale $\sigma$, which is convolved with the image. Fig. 7 shows the behavior of the described algorithm. Then, the upper and lower eyelid boundaries are searched by applying a similar method, which considers the contours as arcuate paths. In the literature, there are many variants of this method. For example, there are algorithms designed to reduce the required computational time [70], or to improve the segmentation accuracy $[60,65]$.

Another important technique used for the estimation of the iris boundaries is based on the Hough transform [31]. The Hough transform permits to isolate the features of a predefined shape within an image. This algorithm can be used for searching different shapes, is robust to noise, and is based on a voting procedure. A well-known iris segmentation technique based on the Hough transform is proposed in [79]. The first step of the method consists in the computation of a binary image that represents the edges of the eye image, obtained by applying a threshold to the magnitude of the gradient of the image. Fig. 8 shows an example of the result obtained by binarizing the magnitude of the gradient image. Then, the Hough transform is performed in order to estimate the circumferences that approximate the inner and outer iris boundaries. Considering circular contours and a set of recovered edge points $\left(x_{j}, y_{j}\right), j=1 \ldots n$, a Hough transform is defined as:

$$
H\left(x_{c}, y_{c}, r\right)=\sum_{j=1}^{N} h\left(x_{j}, y_{j}, x_{c}, y_{c}, r\right)
$$

where:

$$
h\left(x_{j}, y_{j}, x_{c}, y_{c}, r\right)= \begin{cases}1, & \text { if } g\left(x_{j}, y_{j}, x_{c}, y_{c}, r\right)=0 \\ 0, & \text { otherwise }\end{cases}
$$


(a)

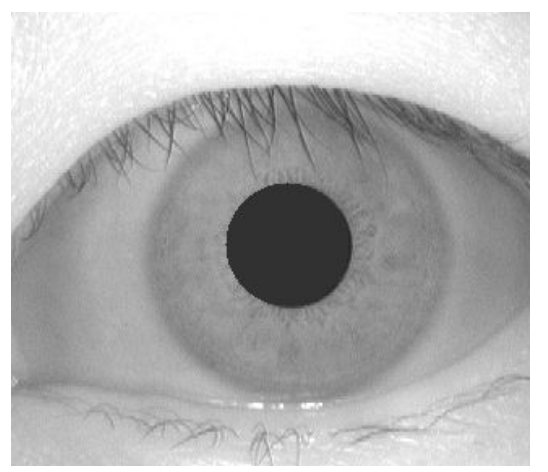

(b)

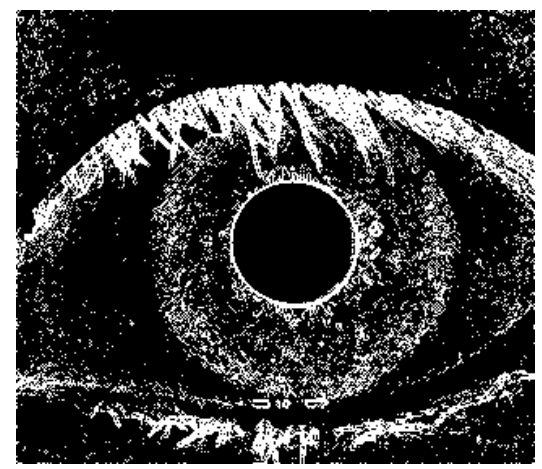

Fig. 8 Example of edge estimation: (a) original image; (b) binary image that describes the edges

with:

$$
g\left(x_{j}, y_{j}, x_{c}, y_{c}, r\right)=\left(x_{j}-x_{c}\right)^{2}+\left(y_{j}-y_{c}\right)^{2}+r^{2} .
$$

Each parameter triple $\left(x_{c}, y_{c}, r\right)$ represents a circumference through an edge point $\left(x_{j}, y_{j}\right), g\left(x_{j}, y_{j}, x_{c}, y_{c}, r\right)=0$. The parameter triple corresponding to the maximum value of $H$ is considered as the result of the method. This result represents the circumference described by the maximum number of points. The last step consists in the estimation of the upper and lower eyelid boundaries. This step is performed similarly, by using the Hough transform to search parameterized parabolic arcs.

In the literature, there are many different iris segmentation methods based on the Hough transform, the main difference consists in the algorithm used for the estimation of the edges. The choice of this algorithm is related to the applicative context. In the case of low contrast images, for example, it is not sufficient to estimate the edges by using an algorithm based on the magnitude of the gradient. The method proposed in [27] first computes the lower contour of the pupil in order to estimate the inner iris boundary, then uses the obtained information to estimate the outer boundary. The last step consists in the refinement of the contours with the application of the circular Hough transform. A method based on the Hough transform and on a robust edge detection strategy is proposed in [55]. This method is designed for the segmentation of noisy iris images and estimates the edges from an image by applying a fuzzy clustering technique on a set of extracted features. There are also methods based on the Hough transform and designed in order to obtain good performances in terms of computational time [75, 73]. A well-known open-source implementation of an iris segmentation method based on the Hough transform and on the Canny edge detector is described in [48]. This software is used as a reference method in many scientific publications.

In the literature, there are also iterative methods for the estimation of the iris boundaries based on operators different from the one proposed in [17]. The method proposed in [10] is based on one of these iterative approaches. A set of candidate center points is selected first. For each of these points, a polar image representing the 
iris is computed. The candidate inner and outer boundaries are then estimated considering the vertical gradient of the obtained polar images. The best fitting boundaries are then estimated by maximizing the function:

$$
\sum_{\theta=1}^{n}\left((n-1)\left\|g_{\theta, r}\right\|-\left(\sum_{\phi=\theta+1}^{n}\left\|g_{\phi, r}-g_{\theta, r}\right\|\right)-g_{\theta, r} / n\right),
$$

where $n$ is the total number of discrete values of the polar variable $\theta$, and $g_{\theta, r}$ is the vertical gradient value. The obtained solution is then refined by iteratively considering the points near to the obtained center as candidate centers. The last step is the approximation of the inner iris boundary to an ellipse and is performed by using a brute-force approach.

The method presented in [22] performs an iterative search of the circumferences that approximate the inner and outer iris boundaries by maximizing the function:

$$
D=\sum_{m}\left(\sum_{k=1}^{5}\left(I_{n, m}-I_{n-k, m}\right)\right)
$$

where $I_{i, j}=I\left(x_{0}+i \Delta_{r} \cos \left(j \Delta_{\theta}\right), y_{0}+i \Delta_{r} \sin \left(j \Delta_{\theta}\right)\right), I(x, y)$ is the image intensity, $\Delta_{\theta}$ is the angle increment, and $\Delta_{r}$ is the radius increment.

The method presented in [71] first estimates the inner iris boundary and then estimates the outer boundary by using an iterative approach. The area of the image used to search the center of the circumference approximating the outer boundary is limited to the pixels that are close to the pupil center. The approximated circumference is defined as a weighted circular integral of local SNR values:

$$
\max _{\left(x_{c}, y_{c}, r\right)} \oint_{\left(x_{c}, y_{c}, r\right)} w_{x_{c}, y_{c}} \frac{\operatorname{SNR}(x, y)}{2 \pi r} d s,
$$

where $\operatorname{SNR}(x, y)$ is the ratio of the local mean to the local standard deviation at the position $(x, y)$, and $w_{i}$ is a weight value, defined as $w_{i}=1-\left(d_{i} / d_{\max }\right)$.

A well-known iris recognition system is proposed in [5]. This system approximates the inner and outer iris boundaries by two circumferences. The first step consists in the localization of the pupil center by using an edge detection technique. Then, the inner and outer iris boundaries are estimated as two concentric circumferences. The radii are estimated by considering the limits described by the edges.

The method proposed in [58] binarizes the image by using the information related to the Fourier spectral density and then estimates the circumferences that describe the inner and outer iris boundaries by using a geometrical approach.

The technique described in $[42,37]$ performs the computation of local statistics (standard deviation and kurtosis) in order to obtain an image that enhances the visibility of the outer iris boundary. This image is then binarized and a circumference representing the iris contour is estimated by iteratively searching the ring containing the maximum number of pixels with intensity equal to one. 


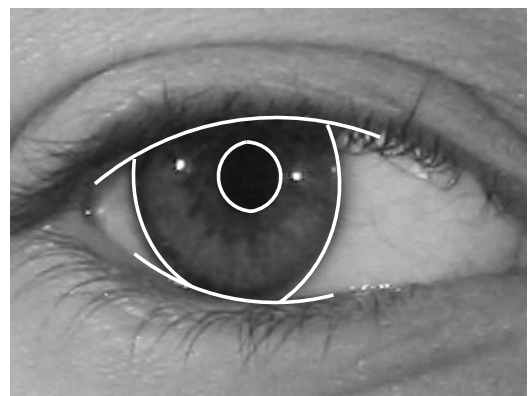

Fig. 9 Example of a result obtained by the methods that approximate the iris boundaries as two ellipses and the eyelashes as two parabolic arcs

\section{Methods based on a-priori models}

The application of circumferences as parametric models to fit the iris boundaries is very common in the literature but, in some specific cases, is not the best solution. The human iris, in fact, is not perfectly circular. Especially for images affected by off-axis problems, the iris boundaries can be better described by using ellipses. For this reason, many segmentation methods in the literature use ellipses for approximating the iris shape. Fig. 9 shows an off-axis iris image segmented by using a method based on the fitting of ellipses.

The estimation of an ellipse is more complex than the estimation of a circle and needs two more parameters. An ellipse is defined by the equation:

$$
a x^{2}+b x y+c y^{2}+d x+e x+f=0
$$

which can also be written as:

$$
a\left(x-C_{x}\right)^{2}+b\left(x-C_{x}\right)\left(y-C_{y}\right)+c\left(y-C_{y}\right)^{2}=1
$$

where $\left(C_{x}, C_{y}\right)$ is the center point, and $a, b, c$ are the shape parameters. The majority of the methods for the estimation of ellipses are based on Equation 9. Usually, the parameters of this equation are estimated starting from the representation $\left(C_{x}, C_{y}, r_{1}, r_{2}, \theta\right)$, where $r_{1}$ and $r_{2}$ are the lengths of the major and minor axis, and $\theta$ is the rotational angle of the major axis.

In the literature, there are different techniques for the approximation of the iris boundaries as ellipses. One of the most known of them is based on the use of the Hough transform. Differently from the method described in [79], this technique does not try to isolate the features related to circular shapes, but considers elliptical paths. The method described in [83] uses the Hough transform in order to refine a first representation of the inner iris boundary. Another method based on this technique is proposed in [35] and uses an improved randomized Hough transform. The edges are first estimated by using the Canny algorithm, then an iterative algorithm is applied. During each iteration, the center point and the outer iris boundary are estimated. The 
iris center is geometrically estimated by computing the intersection of three tangent lines passing for three randomly selected points. Considering these points, the other three parameters describing the ellipse are then obtained by solving a linear system. The obtained result consists in the ellipse that best fits the iris boundary after a certain number of iterations.

Another important method is based on the integro-differential operator proposed in [17]. This method computes the integro-differential operator considering elliptical paths instead of circular paths. Examples of the obtained results are reported in [53].

A different technique for estimating the iris boundaries as two ellipses is the "Starburst" method [64]. This method can be divided in three steps: reference point selection, feature extraction, ellipse fitting, and estimation of the eyelids. The first step consists in the selection of a point near to the pupil center. The pupil area is first estimated by an intensity thresholding task. Then, the reference point is estimated as the centroid of the pupil area. Starting from this reference point, a set of rays in different directions are computed. The gradient is calculated along each ray and the obtained values are used in order to identify the feature points of the inner and outer boundaries. The upper and lower regions of the boundaries are not considered because they can be affected by occlusions. The obtained feature points are then used in order to estimate the ellipses that approximate the inner and outer iris boundaries. The proposed method iteratively estimates an ellipse from a set of five randomly selected points. During each iteration, the correctness of the estimated ellipse is evaluated, and the final result consists in the ellipse computed as the average of the best estimated ellipses. The last step performs the detection of the eyelids by using a snake algorithm.

Another approach based on elliptical shapes is described in [76]. The proposed method uses an active ellipse modeling technique for estimating the outer iris boundary. This method estimates the features by computing two spatial scales of the Petrou-Kittler optimal ramp filters. The fitting ellipse is iteratively estimated by using a simulated annealing algorithm.

The method described in [26] performs a first estimation of the iris area and then approximates the inner and outer iris boundaries by using an ellipse fitting technique based on direct least squares [29].

\section{Methods based on the analysis of local characteristics}

This section describes the methods that perform the segmentation of the iris region by using local image characteristics. Most of these methods segment the eye images by using computational intelligence classifiers.

The method presented in [53] is based on neural classifiers and is designed to work with noisy color iris images, captured in visible light conditions. A modified version of the method is also proposed for performing the segmentation of images captured using infrared light illuminators. The segmentation method is composed 
of two steps: detection of noise-free iris pixels, and parameterization of the iris shape. The first step estimates the pixels appertaining to the sclera and to the iris by using two trained classifiers. The estimation of the area occupied by the sclera is performed by using feedforward neural networks in order to classify every pixel of the eye image. For each pixel, the extracted features are the mean and standard deviation of Haar-like features computed in local regions of different color spaces. Similarly, the iris area is estimated by classifying the pixels with feedforward neural networks. The used features are related to different color spaces and consist in the pixel position, local image saturation, blue chrominance, and the values that represent the proportion of pixels belonging to the sclera in the four directions. The last step is the parameterization of the iris shape. First, the centers of the iris and pupil are roughly estimated, then the inner and outer iris boundaries are extracted in Polar coordinates and parameterized by performing a polynomial regression. The last task is the conversion of the obtained results in Cartesian coordinates.

An approach based on a K-means clustering technique is described in [43, 41]. This method is designed for the segmentation of noisy iris images captured in unconstrained conditions. The method is divisible in these steps: iris detection, region clustering, approximation of the iris boundaries, and detection of the eyelids. The iris detection step checks for the iris pattern in the image and roughly estimates its position, using an Adaboost approach. Then, the K-means clustering technique is applied. The number of clusters is adaptively estimated from the histogram of the eye image. The obtained result is an image in which each gray-level corresponds to a cluster. The subsequent step is the approximation of the iris boundaries. First, the clustered image is treated by using a Canny edge detector in order to enhance the visibility of the iris boundaries. The outer boundary is then obtained by applying an algorithm based on a modified Hough transform, and the inner boundary is then estimated by using the method based on the integro-differential operator presented in [17]. The last step is the detection of the eyelids and is performed by using an integro-differential operator based on parabolic arcs. This algorithm constrains the possible candidate parabolas by using an approach based on the random sample consensus technique (RANSAC).

Another method that uses the K-means clustering for obtaining a first estimation of the iris area is proposed in [26]. This method is designed in order to operate with frame sequences captured in uncooperative situations by using infrared light illuminators. The described approach can be divided in four steps: quality evaluation of the frame, clustering, iris border approximation, and estimation of the eyelids. The quality estimation step searches the presence of specular reflections in order to identify the presence of the iris in the evaluated frame. This task is performed by using an algorithm called quality filter, which is based on a downsampling stage and a high-pass filter. The clustering is then performed, obtaining an image in which the iris and the pupil are described by low intensity values. This step uses the Kmeans clustering technique and principal component analysis (PCA). During the approximation of the iris boundaries, the pupil center is searched, the edges are extracted, and two ellipses representing the inner and outer boundaries are estimated. The pupil center is extracted by considering the obtained rough representation of the 
pupil. The edges are then searched in Polar coordinates by considering the angular derivative of the image. The approximation of the iris boundaries is then performed by using a direct least square fitting of ellipses. The estimation of the eyelids is the last step. A map of the noise present in the region of interest is obtained by computing the local variance of the image. The shape of the eyelids is then approximated by a second order polynomial.

The method proposed in [9] uses neural classifiers in order to segment the eye images without using assumptions related to the circularity of the iris boundaries. Each pixel is classified into three classes (iris, pupil, other) by using multi-layer feedforward neural networks. The used features are related to the distance between the considered pixel and the pupil center, the local intensity, and the orientation of the local area centered in the pixel. A post-processing stage is used to ensure the connectedness and smoothness of the resulting segmentation mask. The work described in [9] presents also a study related to the selection of the most discriminative features.

Support vector machines (SVM) are used by the method described in [62]. This method approximates the inner and outer iris boundaries by two circles. First, the inner boundary is estimated by an algorithm based on the Hough transform. Then, the outer boundary is estimated by using a pixel classifier based on SVM, and refined by applying the Hough transform. The used features are related to the local shape and local gradient.

The method proposed in [59] is based on the graph cut technique, which is also used in many computer vision applications [8]. The iris segmentation method models the eye image as a Markov random field, and uses an energy minimization algorithm based on graph cuts in order to estimate eyelashes, pupil, iris, and background. The iris boundaries are then approximated by two ellipses by using a least squares method.

\section{Approaches based on active contours}

Algorithms based on active contours, or snakes, iteratively adapt the segmented shape to the edges of the image. This class of algorithms is commonly adopted in many computer vision applications.

The method described in $[63,69]$ estimates the iris boundaries by employing an approach based on geodesic active contours. The inner and outer iris boundaries are estimated by using two different techniques. First, the image is binarized using a dynamically estimated threshold value. The obtained binary image is then treated by a bidimensional median filter to reduce the number of candidate pupil areas. In order to correctly detect the shape of the pupil area, a circle fitting procedure is applied on all the candidate regions. The circle with the maximum perimeter is considered as the pupil. The outer iris boundary is estimated by using a method based on geodesic active contours. The idea is to iteratively grow the estimated iris boundary until it matches the real iris contour (Fig. 10). This method considers the relation 
(a)

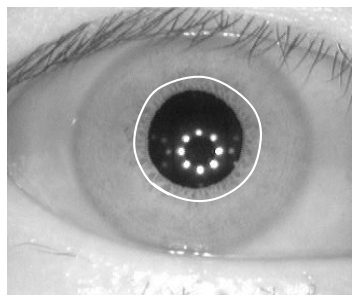

(b)

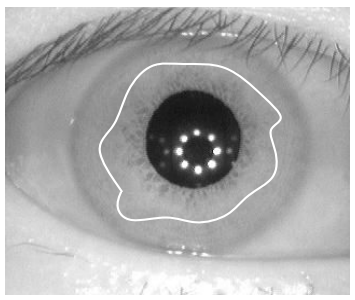

(c)

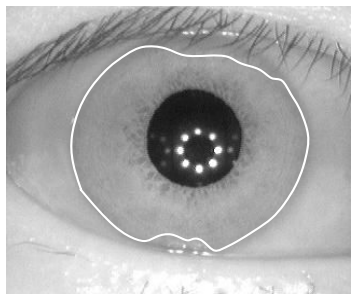

Fig. 10 Example of the evolution of active contours: (a) initial contour; (b) contour after $N$ iterations; (c) contour after $M$ iterations $(M>N)$

between active contours and the geodesics (curves with minimal length). First, an initial approximating curve representing the outer iris boundary is computed as a circumference similar to the inner pupil boundary. During each iteration, this curve evolves towards the iris boundary by considering the Thin Plate Spline energy. The Thin Plate Spline energy is a measure of the distance between the points of the estimated curve and the results obtained by applying a Thin Plate Spline interpolation. The algorithm stops when the energy difference between two subsequently estimated curves is less than a threshold value.

The presence of local spikes is one of the most critical factors for the methods based on active contours. In order to avoid this problem, the method proposed in [82] uses a distance map, called semantic iris contour map, which permits to enhance the visibility of the iris boundaries and to decrease the importance of other details. This map is obtained by computing the gradient image and sigmoid functions.

Another method based on the active contours technique is described in [39]. This method is optimized for the segmentation of the inner pupil boundary.

\section{Hybrid and incremental methods}

This section describes hybrid and incremental methods that perform a first estimation of the iris boundaries and then refine the obtained contours by using different techniques.

The method proposed in [19] describes the iris boundaries without assuming a fixed geometrical shape. This method can be considered as incremental because it first estimates the points of the image appertaining to the iris boundaries, and then refines the inner and outer boundaries by using an approximation technique based on the Fourier series. In order to perform a rough estimation of the iris boundaries, the iris center is estimated first. Using this point as a reference, the image is then converted in Polar coordinates, and the gradient in the radial direction is computed in order to enhance the visibility of the boundaries. The intensity peaks in the resulting image correspond to the points appertaining to the iris boundaries. The vectors 
(a)

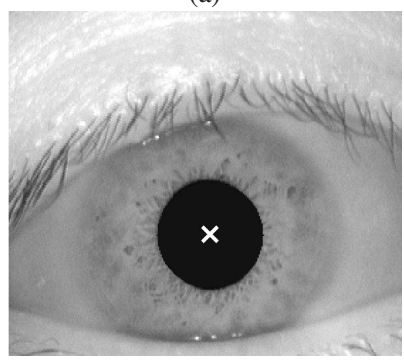

(b)

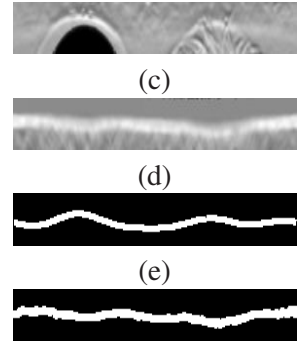

(f)

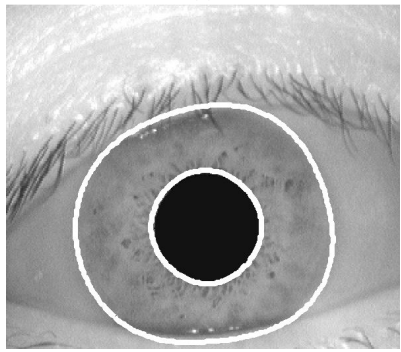

Fig. 11 Example of the results obtained by the Fourier active contours method: (a) input image and iris center; (b) region of the radial gradient image that describes the outer iris boundary in Polar coordinates; (c) region of the radial gradient image that describes the inner iris boundary in Polar coordinates; (d) outer boundary in Polar coordinates; (e) inner boundary in Polar coordinates; (f) inner and outer iris boundaries in Cartesian coordinates

representing the inner and outer iris boundaries are then treated by an approximation algorithm based on the Fourier series. This algorithm considers the edges estimated in Polar coordinates and permits to approximate the shape of the boundaries by polynomials with an arbitrary number of degrees of freedom, in order to reduce the problems related to the presence of noise and occlusions. The obtained function describes a closed curve in Cartesian coordinates. The first step of this approximation algorithm consists in the computation of an arbitrary number $M$ of discrete Fourier coefficients:

$$
C_{k}=\sum_{\theta=0}^{N-1} r_{\theta} e^{-2 \pi i k \theta / N}
$$

where $N$ is the number of regularly spaced angular samples, $r_{\theta}$ is the intensity value at the angle $\theta$ (for $\theta=0 \ldots N-1$ ), $C_{k}$ is a discrete Fourier coefficient (for $k=$ $0 \ldots M-1$ ). The approximated vector of boundary points $R_{\theta}$ (for $\theta=0 \ldots N-1$ ) is obtained as:

$$
R_{\theta}=\frac{1}{N} \sum_{k=0}^{M-1} C_{k} e^{2 \pi i k \theta / N} .
$$

The number of considered coefficients $M$ corresponds to the number of degrees of freedom of the shape. The last step consists in the conversion of the obtained vectors in Cartesian coordinates. Fig. 11 shows an example of the obtained results.

Another incremental approach is described in [25]. The presented method aims to segment noisy iris images captured in unconstrained conditions. This method can be divided in three steps: estimation of the circumferences that approximate the inner and outer iris boundaries, extraction of the edges, regularization of the iris boundaries. First, the centers and radii of the inner and outer iris boundaries are estimated by using a technique similar to the integro-differential operator described in [17]. The refinement of the iris boundaries is then performed by using an algorithm similar to the one proposed in [19]. In order to enhance the visibility of the iris boundaries, the gradient in the radial direction is computed for the two previ- 
(a)

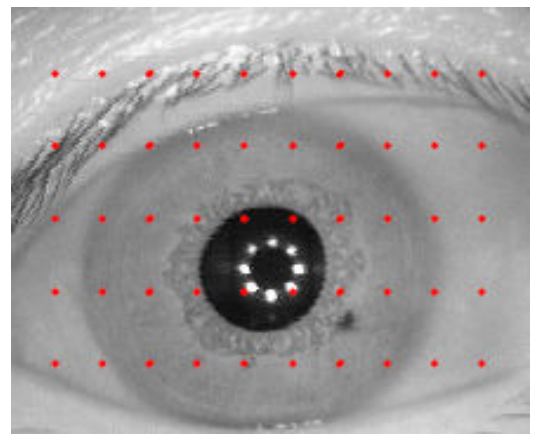

(b)

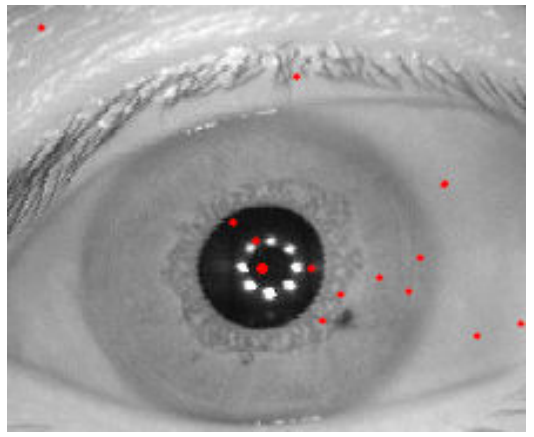

Fig. 12 Graphical representation of the agent-based approach: (a) initial deployment; (b) final positions of the agents. The majority of the agents are accumulated in the central point of the pupil, correctly estimating the pupil center

ously estimated center positions. Two stripe images related to the inner and outer iris boundaries are then computed by applying a conversion in Polar coordinates and considering the values of the previously estimated radii. The candidate points belonging to the iris boundaries are then searched considering the maximum intensity values for each column of the two strip images. The last step is the regularization of the iris boundaries, obtained by applying an outlier removal algorithm. This algorithm achieves the selection of continuous intervals of the boundaries by using a double hysteresis thresholding analysis. The obtained boundaries are then fitted by using the approximation method based on the Fourier series [19] and converted in Cartesian coordinates.

An incremental method based on computational intelligence techniques is described in [23]. This method first estimates the iris position by using a multiple agent approach, and then performs a refinement of the iris boundaries by applying an algorithm based on multiple points of views. The goal of the multiple agent approach is to estimate the position of the pupil center. First, a set of agents are initialized at different positions in the input image (Fig. 12a). Each agent collects information related to the local intensity pattern and then moves its position according to the estimated local properties. After few iterations, the population tends to spread and then concentrates in the inner portion of the pupil (Fig. 12b). The method stops when a sufficient number of agents are situated in a circular area, or when the maximum number of iterations is reached. The agents are based on neural networks [24]. Each agent performs a feature extraction task and a decision task. During the feature extraction, the radial gradient image centered in the agent position is computed and converted in Polar coordinates. For each angular value $\theta$ of the computed image, the coordinates of the point with the maximum intensity value are estimated and stored in a vector $Y$. Then, a polynomial is fitted on the values of $Y$, and the mean square error of the regression is computed. The polynomial coefficients and the mean squared error value are the inputs of the decision step. This step is based 
(a)

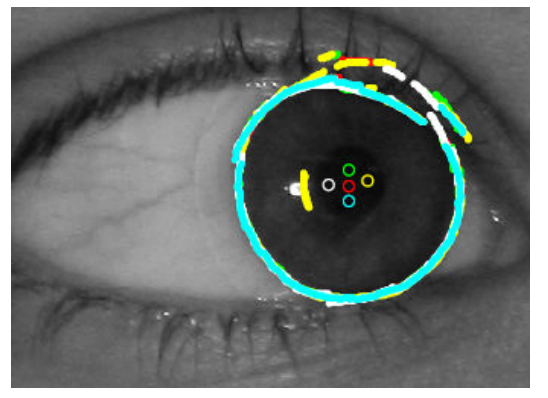

(b)

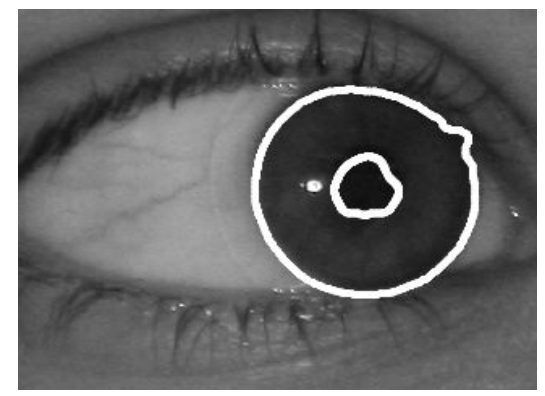

Fig. 13 Example of results obtained by the multiple boundary refinement method: (a) multiple boundaries computer using different observation points; (b) merged boundaries

on a trained feedforward neural network, which returns the displacements in the $x$ and $y$ directions with respect to the pupil center. The inner and outer iris boundaries are then estimated by using an approach that considers multiple observation points. The coordinates of these points are computed in the proximity of the previously estimated pupil center. For each observation point, a vector describing the candidate iris boundary is estimated. During this task, a radial gradient image is computed and converted in Polar coordinates. The maximum intensity value of each column of this image is then considered as a candidate edge point. All the obtained vectors of candidate boundary points are then translated around a common pivot point. The last task consists in the merging of the obtained information. During this task, a binary image describing the candidate boundary coordinates estimated by every observation point is computed. In order to remove the presence of noise, morphological operations are applied. The iris boundaries are then estimated and refined by using the approximation method based on the Fourier series [19]. An example of the obtained results is shown in Fig. 13.

The method proposed in [34] is based on a different incremental approach. This method can be divided in four steps: removal of specular reflections, rough localization of the iris area, estimation of the iris boundaries, and approximation of the eyelids. The first step permits to reduce possible segmentation errors caused by strong reflection areas. Specular reflections are removed by applying an algorithm that eliminates the intensity peaks of the image and then interpolates the missing values. The iris detection is then performed by using a trained Adaboost-cascade classifier [77]. This algorithm identifies the presence of the iris in the image and performs a rough estimation of the image area occupied by the iris. The algorithm performs a five-layer validation and is based on Haar-like features extracted from local areas of different sizes. The estimation of the inner and outer iris boundaries is then computed by using the proposed "pulling \& pushing" algorithm [32]. This algorithm estimates the iris boundaries without approximating their shape with a-priori models. This process is based on physical laws and aims to search the equilibrium position between massless springs (Fig. 14). The algorithm iteratively improves the 
(a)

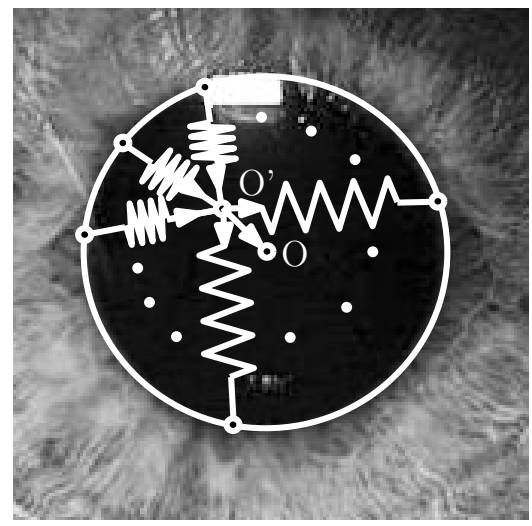

(b)

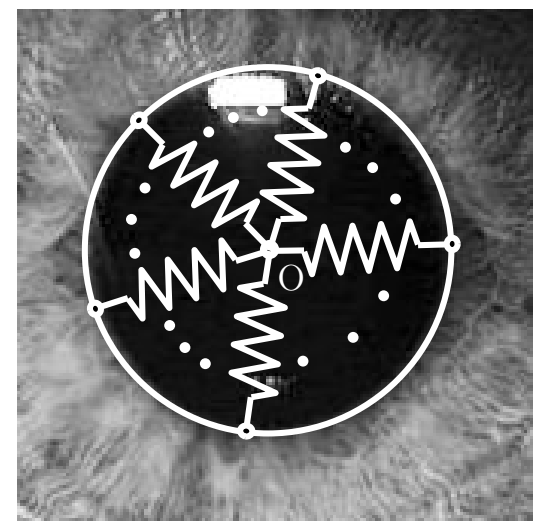

Fig. 14 Visual representation of the "pulling \& pushing" algorithm: (a) the candidate center $O^{\prime}$ is pushed towards the real center $O$ by the forces exerted by the springs; (b) the equilibrium position is reached

shape of the iris boundary. During each iteration, the edges are estimated in Polar coordinates related to a temporary center position, the spikes are removed, and the parameters describing an approximating circumference are then obtained by computing the equilibrium point. The segments between the edges and the temporary center position are considered as springs. After each iteration, the center position and radius value are modified. The algorithm terminates when the equilibrium is obtained, or when the maximum number of iterations is reached. After this iterative process, the obtained vector of edges is fitted by using a technique based on the cubic smoothing spline interpolation, obtaining the final shape of the boundary. The last step consists in the eyelid localization. The method searches the best fitting of the eyelids by using statistically established eyelid curvature models.

The method presented in [83] uses an incremental approach in order to estimate the inner and outer iris boundaries as two ellipses. This method can be divided in four steps: preprocessing, pupil localization, inner boundary estimation, and outer boundary estimation. The preprocessing step estimates the specular reflections by using a thresholding approach, removes the estimated areas, and interpolates their intensity values. The pupil localization obtains a first estimation of the pupil area and uses an algorithm based on the circular Hough transform. The inner iris boundary is then estimated by applying an ellipse fitting technique. The outer iris boundary is estimated using a method similar to the one proposed in [19], but designed for elliptical spaces. The elliptical rotated space used for searching the outer iris boundary is defined as:

$$
\begin{aligned}
\left(r, \phi, x_{i 0}, y_{i 0}\right): & \left(\left(x-x_{i 0}\right) \cos \phi+\left(y-y_{i 0}\right) \sin \phi\right)^{2} /(a r)^{2}+ \\
& \left(-\left(x-x_{i 0}\right) \sin \phi+\left(y-y_{p 0}\right) \cos \phi\right)^{2} /(b r)^{2}=1,
\end{aligned}
$$


where $\left(x_{i 0}, x_{i 0}\right)$ is the pupil center, $a$ and $b$ are the parameters of the ellipse fitted into the pupil, $\phi$ is the angle of the principal axis, and $r$ is the scale factor. The iris boundary is then obtained by a maximization:

$$
\max _{r, x_{i 0}, y_{i 0}}\left[\frac{1}{L\left(r, x_{i 0}, y_{i 0}\right)} \oint_{B}\left(r, x_{i 0}, y_{i 0}\right) F(\nabla(I(x, y))) d s\right],
$$

where $B\left(r, x_{i 0}, y_{i 0}\right)$ is the considered portion of the elliptical boundary, $L\left(r, x_{i 0}, y_{i 0}\right)$ is the number of elements of $B\left(r, x_{i 0}, y_{i 0}\right)$, and $F(\nabla(I(x, y)))$ is a function of the gradient $\nabla(I(x, y))$. In order to simplify the estimation of the edges and the computation of the fitting ellipse, this task is performed by first converting the image in a new coordinate system. This conversion permits to obtain images similar to the polar representation used in [19]. The mapping between the considered ellipse and a circle is defined as:

$$
\left\{\begin{array}{l}
\left(x-x_{i 0}\right) \cos \phi+\left(y-y_{i 0}\right) \sin \phi=\operatorname{arcos} \theta^{\prime} \\
-\left(x-x_{i 0}\right) \sin \phi+\left(y-y_{i 0}\right) \cos \phi=b r \sin \theta^{\prime}
\end{array}\right.
$$

where $\theta^{\prime}=\left(\theta-\phi^{\prime}\right)$, and $\phi^{\prime}=\arctan (a / b(\phi))$.

\section{Other methods}

This section reviews the methods present in the literature that do not fall in any of the previously described classes.

The method proposed in [14] performs the iris recognition using iris images captured by a special device composed by two CCD sensors, which simultaneously capture an infrared image and a RGB color image. This system uses the color image in order to better perform the segmentation step. The inner and outer iris boundaries are estimated by using two different algorithms. The inner iris boundary is estimated by considering only the infrared image. First, a rough representation of the pupil area is obtained by using a method based on the search of dark pixels. Then, the outer iris boundary is estimated by using a direct least square fitting of the ellipse [29]. The outer iris boundary estimation considers the information related to both the infrared image and the RGB image. For each color channel, the edges are extracted by using the Canny algorithm. A trained classifier is then adopted in order to remove the noisy edge points. The outer iris boundary is finally estimated as a circumference by applying the RANSAC technique. An algorithm similar to the one proposed for the estimation of the outer iris boundary is then used for estimating the shape of the eyelids. This algorithm is based on the RANSAC technique for the searching of parabolic shapes.

Another example of approach that cannot be included in the previously described classes is presented in [46]. The proposed method achieves the iris segmentation by using mathematical morphological operators. This method is composed by five steps: channel selection, reflection removal, outer boundary estimation, and inner 
boundary estimation. First, the RGB channel with the maximum entropy of the histogram is selected in order to obtain the image with the best contrast. Then, an opening operator and a thresholding task are applied to estimate the reflections. The first task of the outer boundary estimation consists in searching the iris center. This task is performed without considering the iris as a circular object. Two close-hole operators and image inversions are computed. The point with the maximum difference value between the original image and the obtained image is chosen as the corresponding center. Then, a first approximation of the outer boundary is performed. During this task, the eye image is converted in Polar coordinates, the visibility of the boundary is improved by applying a morphological multiscale gradient, and an anisotropic averaging filter is applied in order to reduce the presence of noise. The outer iris boundary is then estimated as the shorter continuous path that follows the transition between the iris and sclera. The last task of the outer boundary estimation is the suppression of the eyelids and eyelashes. This task cuts the higher region of the previously segmented area by using a line, which is obtained by the evaluation of the intensity values. The last step is the inner boundary estimation. This step is similar to the outer boundary estimation but the searching of the pupil center and the estimation of the edges are limited to the previously estimated region of interest.

The method proposed in [74] is designed for the segmentation of noisy iris images captured in unconstrained conditions and with visible light illumination. Similarly to the technique described in [34], this method is divisible in four different steps: removal of the specular reflections, rough iris area estimation, approximation of the inner and outer iris boundaries, and eyelid localization. The removal of the specular reflections is performed by using the algorithm described in [34]. The second step aims to obtain a rough estimation of the iris area and to identify the non-iris regions (e.g. eyelashes and eyebrows). This step is performed by using a clustering technique based on two different algorithms. The first one creates the clusters by using the intensity information and then expands the area of these clusters by using an iterative technique. The second algorithm performs a semantic refinement in order to identify the candidate iris region and the non-iris regions. This algorithm tries to label the different areas of the image by using the information related to the shape, intensity and position of each clustered region. The estimation of the inner and outer iris boundaries is performed by using an algorithm based on the integro-differential operator presented in [17]. This algorithm aims to speed up the segmentation based on the integro-differential operator and to limit the problems related to the presence of local optima in the image. The algorithm iteratively searches the shortest path by using a technique that considers the results obtained applying the integrodifferential operator in a constellation of near points. The last task performed during this step consists in the boundary refinement, which is based on an algorithm that uses intensity statistics. The last step consists in the eyelid localization. The used algorithm is similar to the one presented in [34] and searches the best fitting of the eyelids based on a set of statistically established eyelid curvature models. 


\section{Reflections and occlusions}

This sections reviews the methods especially designed to remove reflections, shadows, and occlusions (such as eyelids and eyelashes) from the iris image. Some examples of occlusions that can typically be found in iris images are shown in Fig. 4. First, reflection detection methods are presented, and then methods for the segmentation of eyelids and eyelashes are described.

The reflection detection methods can be divided in methods based on the image intensity thresholding and methods based on the frequency analysis.

An approach based on a thresholding algorithm followed by an inpainting procedure based on the derivative of the image is proposed in [83]. Another method based on a thresholding technique is described in [63]. The method proposed in [65] uses an adaptive procedure for the computation of the segmentation threshold, defined as a fixed proportion between the average and maximum intensity values of the image. A morphological processing is then applied to refine the segmentation of the reflections.

The method described in [26] detects the iris regions affected by specular reflections by using an high-pass filter. The frequency analysis is particularly distinctive for the specular reflection estimation because small bright regions in a dark area have high frequency values.

A distinction between small and large reflections is made in [25]. Small reflections are detected by thresholding the regions with high local variance values, while large reflections are detected by considering the intensity levels greater than a fixed threshold.

The majority of the methods for the segmentation of eyelids and eyelashes are based on edge detectors, local variance thresholding, or intensity thresholding.

The approach described in [25] removes the eyelashes by considering separable and non-separable eyelashes. Separable eyelashes are detected by applying a Gabor filter tuned to extract the distinctive features of eyelashes. Non-separable eyelashes are segmented by thresholding the regions with the greatest local variance values. The computed occlusion masks are then merged by using a region-growing approach.

The method presented in $[33,34,74]$ first estimates the boundaries of the eyelids, and then removes eyelashes and reflections. The positions of the eyelids are first estimated by considering the results of a rough searching of the eyelashes. Since the shape of many eyelashes is similar to a thin and vertical line, the used algorithm performs the eyelid localization by first using a 1-D horizontal rank filter to remove separable eyelashes. An eyelid edge map is then computed by performing an edge detection in the vertical direction. Then, a shape similarity approach is used to remove noisy edge points and to approximate the shape of the eyelids. The obtained result consists in two parabolic arcs. The detection of eyelashes and shadows is based on a local intensity threshold algorithm. The resulting areas are then connected to the upper eyelid.

The method described in [80] uses an approach based on the computation of a set of possible models that describe the position of the eyelids and eyelashes in the 
image. After the iris location and normalization steps, the best model is searched in the image and the corresponding region is removed from the segmented image.

An approach based on morphological operators is described in [81], and starts from the assumption that pixels belonging to eyelashes form a connected region, since they all start from the eyelid. The pixel with the lowest intensity value is then chosen as the starting point for a region growing process. New pixels belonging to the region are attached to the growing region if the absolute difference between their intensities is less than a fixed threshold.

The method described in [3] uses a wavelet transformation to detect and remove eyelashes regions from iris acquisitions. This method is based on the fact that eyelashes are particularly visible in the vertical and diagonal coefficients of wavelet transforms. These coefficients are then set to zero and the inverse wavelet transformation is used to reconstruct the image. The filtered image is then subtracted from the original image to obtain an image with enhanced eyelashes. The Hough transform is then applied to the enhanced image for detecting the curves describing the eyelashes. The points belonging to the eyelashes are then classified using a previously trained multi-layer perceptron network, which outputs the probability of each pixel to belong to an eyelash region.

A method that uses a filtering approach for the detection of eyelids is described in [40]. After the estimation of the iris boundaries, a searching area for the eyelid border is defined according to two cross points, computed from the discontinuities found in the boundary. Then, the region is filtered by using masks designed to enhance the eyelid border. The candidate points are then fitted by using the Hough transform. Eyelashes are then searched starting from the computed eyelid regions. Non-separable eyelashes are searched by using a local window and by thresholding the intensity and standard deviation values. Separable eyelashes are searched by performing an image filtering with a specially designed kernel in order to enhance separate thin lines.

\section{Performance evaluation}

\subsection{Techniques for performance evaluation}

During the design of a biometric segmentation method, the evaluation of the accuracy is a fundamental step, which can be achieved by following two different strategies:

- pixel level evaluation;

- system level evaluation.

The pixel level evaluation can be considered as a comparison between the binary image obtained by the segmentation method and a corresponding manually segmented image. In order to properly compare the performances of different methods, 
(a)

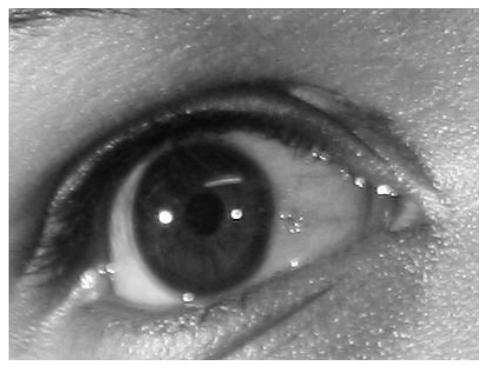

(c)

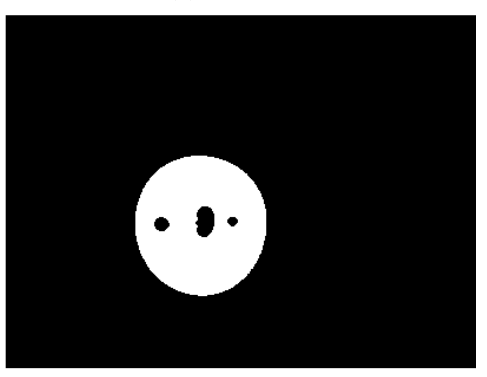

(b)

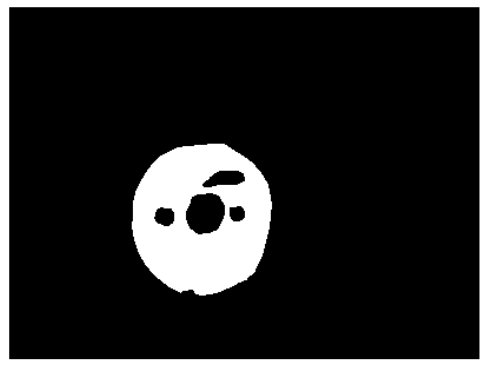

(d)

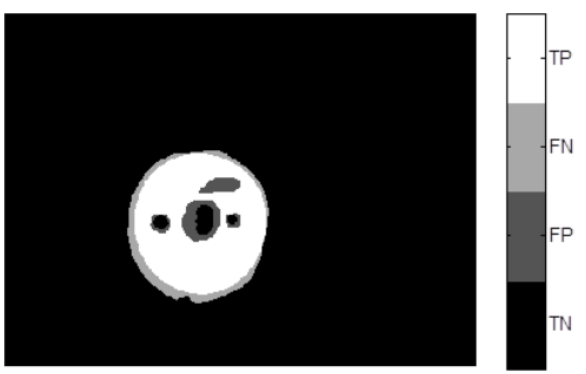

Fig. 15 Visual examples of the proposed figures of merit: (a) eye image $I_{i}$; (b) manually classified binary iris image $C_{i}$; (c) output of the segmentation algorithm $O_{i}$; (d) classified image areas $\left(T P_{i}\right.$, $\left.F N_{i}, F P_{i}, T N_{i}\right)$

it is necessary to define common figures of merit. Considering an image $I_{i}$, the output of the segmentation algorithm $O_{i}$, and the manually classified binary iris image $C_{i}$, it is possible to use these definitions:

- Positives $\left(P_{i}\right)$ - the pixels belonging to the iris in the image $C_{i}$;

- Negatives $\left(N_{i}\right)$ - the pixels belonging to the background in the image $C_{i}$;

- True positives $\left(T P_{i}\right)$ - the number of pixels correctly classified as positive in $O_{i}$;

- True negatives $\left(T N_{i}\right)$ - the number of pixels correctly classified as negative in $O_{i}$;

- False positives $\left(F P_{i}\right)$ - also known as type I error, is the number of pixels classified as positive in $O_{i}$, but are negative in $C_{i}$;

- False negatives $\left(F N_{i}\right)$ - also known as type II error, is the number of pixels classified as negative in $O_{i}$, but are positive in $C_{i}$;

- Sensitivity $\left(\right.$ Sens $\left._{i}\right)$ - the probability of correctly classifying the pixels describing the iris, equal to: $T P_{i} /\left(T P_{i}+F N_{i}\right)$;

- Specificity $\left(\operatorname{Spec}_{i}\right)$ - the probability of correctly classifying the pixels describing the background, equal to: $T N_{i} /\left(T N_{i}+F P_{i}\right)$;

- Classification error $\left(E_{i}\right)$ - is the number of misclassified pixels, defined as $E_{i}=$ $F P_{i}+F N_{i}$.

Fig. 15 shows a visual example of the proposed figures of merit. 
It is possible to compute the error rates in terms of the number of columns $c$ and the number of rows $r$. For example, the classification error rate $E R_{i}$ can be computed as:

$$
E R_{i}=\frac{E_{i}}{(r \times c)} .
$$

It is also possible to extend the previous definitions by considering a dataset composed by $n$ images and averaging the results obtained for every image $I_{i}$. For example, the average classification error rate $\bar{E}$ can be computed as:

$$
\bar{E}=\frac{1}{n} \sum_{i} E R_{i}
$$

The system level evaluation of an iris segmentation method is related to the use of the designed algorithm in a complete biometric recognition system. This technique is necessary for testing the effective accuracy improvement in a real applicative context. In many situations, it is also difficult or impossible to use a sufficient number of manually classified binary iris images to perform a significant pixel level evaluation.

In order to evaluate the obtained accuracy on a test dataset, it is necessary to consider the obtained distributions related to genuines and impostors. One of the most used metrics consists in the computation of the receiver operating characteristic (ROC). Some important numerical indexes that should be evaluated are the equal error rate (EER), the false match rate (FMR), and the false non-match rate (FNMR) [30].

\subsection{Public datasets}

The use of public datasets for the performance evaluation allows to make a comparison of the performance obtained by the designed method and the performances of the techniques already available in the literature. Moreover, it is not necessary to spend time and resources in the collection of a big database of biometric samples.

One of the most used datasets in scientific articles is the CASIA Iris Database [36] proposed by the Institute of Automation of the Chinese Academy of Sciences (CASIA). There are four progressive versions of this dataset. The last version is the CASIA Iris Image Database Version 4.0 and is composed by 6 sets of images:

- CASIA-Iris-Interval: close-up iris images captured using a CCD and a circular near infrared LED array developed by the CASIA.

- CASIA-Iris-Lamp: images collected using a hand-held iris sensor, and featuring problems related to the pupil movements under different illuminations.

- CASIA-Iris-Twins: images captured from 100 pairs of twins.

- CASIA-Iris-Distance: dual eyes images captured using a multi-biometric device. The distance between the user and the sensor is around $3 \mathrm{~m}$.

- CASIA-Iris-Thousand: 20,000 iris images captured from 1,000 subjects by using a commercial device. 
- CASIA-Iris-Syn: 10,000 synthesized iris images.

The CASIA Iris Image Database for Testing Version 1.0 (or IR-TestV1), also publicly available, is composed by 10,000 iris images, captured from 1,000 subjects by using a commercial sensor.

Another important dataset is the ND-IRIS-0405 iris database [6], which includes the ICE 2005 [49] and ICE 2006 [52] subsets. This dataset was collected by the University of Notre Dame and is composed by iris images captured using a commercial sensor in NIR light conditions.

The datasets UBIRIS.v1 [54] and UBIRIS.v2 [57] are composed by noisy images captured at-a-distance and under visible light. A subset of the UBIRIS.v2 dataset was used for the NICE.I [56] competition. For each image of this subset, a manually classified binary iris image is available.

Another important collection of iris images is contained in the multibiometric dataset BIOSEC [28].

Other useful datasets, released by the West Virginia University [78], are the "WVU: Multimodal Biometric Dataset Collection" [15], the "WVU: Off Axis/Angle Iris Dataset Collection", the "WVU: Synthetic Iris Dataset Collection" [68, 84], and the "Clarkson University: Quality-Face/Iris Research Ensemble (Q-FIRE)" [67, 66].

\subsection{Recent results}

At the best of our knowledge, the most recent comparison of iris segmentation methods performing pixel level evaluations is related to the NICE.I competition [56]. Other recent results, in fact, are usually related to system level evaluations.

Table 1 shows the results obtained by the finalist methods of the NICE.I competition. These results refer to the accuracy of segmentation methods on a set of 500 images selected from the dataset UBIRIS.v2. The performances are related to pixel level evaluations and the error values were obtained by using the Equation 16.

One of the most important problems of the images used for the NICE.I competition is the presence of wide reflections. For this reason, most of the more accurate segmentation methods include a reflection removal step before the actual estimation of the iris boundaries.

Other recent results are reported in [53]. These results were obtained by using the Equation 16. The compared methods are an algorithm that approximates the iris boundaries as ellipses by using the integro-differential operator proposed in [17], an implementation of the method proposed in [19], the technique described in [74], and the iris segmentation method presented in [53]. The used datasets are the UBIRIS.v2, 500 iris images cropped from the FRGC face database [50], 500 iris images cropped from the FERET face database [51], and 500 iris images of the ICE-2006 database. Table 2 summarizes the obtained results in terms of accuracy and computational time.

The work described in [34] compares the accuracy of the proposed segmentation method with the results obtained by the segmentation algorithms presented in [17], 
Table 1 Results of the NICE.I competition

\begin{tabular}{|c|c|c|c|c|}
\hline Rank & Authors & Paper title & Reference & Error \\
\hline 1 & Tan et al. & $\begin{array}{l}\text { Efficient and robust segmentation of noisy iris } \\
\text { images for non-cooperative iris recognition }\end{array}$ & [74] & 0.0131 \\
\hline 2 & Sankowski et al. & $\begin{array}{l}\text { Reliable algorithm for iris segmentation in eye } \\
\text { image }\end{array}$ & [65] & 0.0162 \\
\hline 3 & Almeida & $\begin{array}{l}\text { A knowledge-based approach to the iris seg- } \\
\text { mentation problem }\end{array}$ & [4] & 0.0180 \\
\hline 4 & Li et al. & $\begin{array}{l}\text { Robust and accurate iris segmentation in very } \\
\text { noisy iris images }\end{array}$ & [43] & 0.0224 \\
\hline 5 & Jeong et al. & $\begin{array}{l}\text { A new iris segmentation method for non-ideal } \\
\text { iris images }\end{array}$ & [40] & 0.0282 \\
\hline 6 & Chen et al. & $\begin{array}{l}\text { A highly accurate and computationally efficient } \\
\text { approach for unconstrained iris segmentation }\end{array}$ & [11] & 0.0297 \\
\hline 7 & Donida Labati, Scotti & $\begin{array}{l}\text { Noisy iris segmentation with boundary regular- } \\
\text { ization and reflections removal }\end{array}$ & [25] & 0.0301 \\
\hline 8 & Luengo-Oroz et al. & $\begin{array}{l}\text { Robust iris segmentation on uncalibrated noisy } \\
\text { images using mathematical morphology }\end{array}$ & [46] & 0.0305 \\
\hline
\end{tabular}

Table 2 Results reported in [53]

\begin{tabular}{llcccc}
\hline \multirow{2}{*}{ Method } & & \multicolumn{4}{c}{ Dataset } \\
\hline Modified Daugman's method [17] & Error (\%) & 13.97 & 15.10 & 16.83 & 2.39 \\
& Time (s) & 2.73 & 2.73 & 2.73 & 2.73 \\
Fourier active contours [19] & Error (\%) & 6.20 & 8.10 & 9.96 & 1.79 \\
& Time (s) & 3.90 & 4.66 & 4.69 & 4.41 \\
T. Tan et al. [74] & Error (\%) & 1.72 & 4.20 & 5.02 & 2.26 \\
& Time (s) & 5.08 & 5.03 & 5.01 & 4.80 \\
H. Proença [53] & Error (\%) & 1.87 & 4.33 & 4.61 & 2.66 \\
& Time (s) & 0.78 & 0.78 & 0.78 & 0.70 \\
\hline
\end{tabular}

Table 3 Results reported in [34]

\begin{tabular}{llcc}
\hline Method & Performance & ICE 2005 - Left CASIA-IrisV3-Lamp \\
\hline R. Wildes [79] & EER (\%) & 0.64 & 1.05 \\
J. Daugman [17] & EER (\%) & 0.58 & 0.86 \\
Z. He, T. Tan, Z. Sun, X. Qiu. [34] & EER (\%) & 0.53 & 0.75 \\
\hline
\end{tabular}

and in [79]. The biometric recognition is performed by using a method based on ordinal measure filters [72]. Table 3 summarizes the obtained results.

The results reported in [83] are related to the biometric recognition method described in [48]. The segmentation method proposed in [83] is compared with the iris segmentation algorithm described in [48]. Table 4 reports the obtained accuracy obtained by the evaluated methods. 
Table 4 Results reported in [83]

\begin{tabular}{llcc}
\hline Method & Performance & \multicolumn{2}{c}{ Dataset } \\
\hline L. Masek [48] & FRR(\%) @ FAR $=0.1 \%$ & 85.50 & 85.45 \\
J. Zuo, N. A. Schmid [83] & FRR(\%) @ FAR =0.1\% & 2.05 & 3.15 \\
\hline
\end{tabular}

\section{Summary}

Iris recognition systems are considered as the most accurate biometric recognition techniques. One of the most critical tasks of the iris recognition process is the extraction of the area occupied by the iris pattern in eye/face images. An incorrect estimation of this area, in fact, can result in erroneously recognitions. This task, commonly called iris segmentation, has to deal with the fact that the iris region of the eye is a relatively small area, wet and constantly in motion due to involuntary eye movements. Moreover, reflections and occlusions caused by eyelids and eyelashes can be present in the captured images. These problems are more important for the images captured in non-ideal conditions, such as unconstrained, on-the-move, or non-collaborative setups.

The iris segmentation is usually performed in two steps: first the inner and outer boundaries of the iris region are estimated, then occlusions and reflections are detected and removed. It is possible to divide the methods for the estimation of the inner and outer iris boundaries in six classes: methods based on circumferences, methods based on a-priori models, methods based on the analysis of local characteristics, active contours methods, hybrid and incremental methods, methods that do not fall in any of the above mentioned classes.

The methods based on the approximation of the iris boundaries by two circumferences contains well-known methods for iris segmentation, such as algorithms based on an integro-differential operator, and on the Hough transform. The methods based on a-priori models assume a parametric model for approximating the shape of the iris. A circular model can define with a good accuracy a frontal and ideal acquisition, but unconstrained or off-axis images are better modeled by using, for example, an elliptical model. Usually, methods based on a-priori models perform the iris segmentation by searching the edges appertaining to the iris boundaries. However, in the case of degraded or noisy iris images, it can be necessary to use more information in order to obtain accurate results. For this reason, the approaches based on the analysis of local features consider the information related to the iris texture. Moreover, many of these methods do not make any assumptions about the iris shape. Also the methods based on active contours do not perform assumptions regarding the iris shape. These methods combine an iterative contour growing with shape constraints, in order to better match the boundaries of the iris without introducing noise errors due to local image variations. Differently, the hybrid and incremental methods combine techniques from different classes, performing the iris segmentation in an incremental way. Other methods are not easily classifiable, such as a method designed for a special biometric device, a method that performs the iris segmenta- 
tion by using morphological operators, and a method that combines different new algorithms.

The methods for the detection of occlusions and reflections can be based on different techniques, such as thresholding, edge detection, frequency filtering, and wavelet transforms.

In order to properly evaluate and compare the performances of iris segmentation methods, it is necessary to use common techniques and figures of merit present in the literature. Also the adoption of public image datasets is very important since it allows to fairly compare the performances of different methods on the same data with the advantage to reduce the efforts necessary to collect new biometric samples.

Recent results obtained on public datasets show a good accuracy also for images captured in in non-ideal conditions. However, further studies are necessary in order to obtain applicable results also for images captured in completely unconstrained conditions.

\section{References}

1. International Biometrics Group. http://www.ibgweb.com

2. ISO/IEC Standard 19794-6. Information technology - biometric data interchange formats, part 6: Iris image data. Tech. rep., International Standards Organization (2005)

3. Aligholizadeh, M.J., Javadi, S., Sabbaghi-Nadooshan, R., Kangarloo, K.: An effective method for eyelashes segmentation using wavelet transform. In: 2011 International Conference on Biometrics and Kansei Engineering (ICBAKE), pp. 185 - 188 (2011)

4. de Almeida, P.: A knowledge-based approach to the iris segmentation problem. Image Vision Comput. 28(2), 238 - 245 (2010)

5. Boles, W., Boashash, B.: A human identification technique using images of the iris and wavelet transform. IEEE Trans. Signal Process. 46(4), 1185 - 1188 (1998)

6. Bowyer, K.W., Flynn, P.J.: The ND-IRIS-0405 Iris Image Dataset. http://www.nd.edu/ cvrl/papers/ND-Iris-0405.pdf

7. Bowyer, K.W., Hollingsworth, K., Flynn, P.J.: Image understanding for iris biometrics: A survey. Comput. Vis. Image Underst. 110, 281 - 307 (2008)

8. Boykov, Y., Veksler, O.: Graph Cuts in Vision and Graphics: Theories and Applications. In: N. Paragios, Y. Chen, O. Faugeras (eds.) Handbook of Mathematical Models in Computer Vision, chap. 5, pp. 79 - 6. Springer US, New York (2006)

9. Broussard, R., Kennell, L., Soldan, D., Ives, R.: Using artificial neural networks and feature saliency techniques for improved iris segmentation. In: International Joint Conference on Neural Networks (IJCNN 2007), pp. 1283 - 1288 (2007)

10. Camus, T., Wildes, R.: Reliable and fast eye finding in close-up images. In: International Conference on Pattern Recognition, vol. 1, pp. 389 - 394 (2002)

11. Chen, Y., Adjouadi, M., Han, C., Wang, J., Barreto, A., Rishe, N., Andrian, J.: A highly accurate and computationally efficient approach for unconstrained iris segmentation. Image Vision Comput. 28, $261-269$ (2010)

12. Cho, D.H., Park, K.R., Rhee, D.W.: Real-time iris localization for iris recognition in cellular phone. International Conference on Software Engineering, Artificial Intelligence, Networking and Parallel/Distributed Computing, \& International Workshop on Self-Assembling Wireless Networks pp. $254-259$ (2005)

13. Cho, D.H., Park, K.R., Rhee, D.W., Kim, Y., Yang, J.: Pupil and iris localization for iris recognition in mobile phones. In: Seventh ACIS International Conference on Software Engineering, Artificial Intelligence, Networking, and Parallel/Distributed Computing, pp. 197 - 201 (2006) 
14. Chou, C.T., Shih, S.W., Chen, W.S., Cheng, V., Chen, D.Y.: Non-orthogonal view iris recognition system. IEEE Trans. Circuits Syst. Video Technol. 20(3), 417 - 430 (2010)

15. Crihalmeanu, S., Ross, A., Schuckers, S., Hornak, L.: A Protocol for Multibiometric Data Acquisition, Storage and Dissemination. Tech. rep., WVU, Lane Department of Computer Science and Electrical Engineering (2007)

16. Daugman, J.: Statistical richness of visual phase information: Update on recognizing persons by iris patterns. Int. J. Comput. Vision 45, 25 - 38 (2001)

17. Daugman, J.: How iris recognition works. IEEE Trans. Circuits Syst. Video Technol. 14, 21 $30(2002)$

18. Daugman, J.: Results from 200 billion iris cross-comparisons. Tech. Rep. UCAM-CL-TR-635, University of Cambridge, Computer Laboratory (2005). http://www.cl.cam.ac.uk/techreports/UCAM-CL-TR-635.pdf

19. Daugman, J.: New methods in iris recognition. IEEE Trans. Syst., Man, Cybern. B, Cybern. 37(5), $1167-1175$ (2007)

20. Daugman, J.: Iris recognition at airports and border-crossings. In: S.Z. Li, A.K. Jain (eds.) Encyclopedia of Biometrics, pp. $819-825$ (2009)

21. Daugman, J., Malhas, I.: Iris recognition border-crossing system in the UAE. International Airport Rev. 2 (2004)

22. de Martin-Roche, D., Sanchez-Avila, C., Sanchez-Reillo, R.: Iris recognition for biometric identification using dyadic wavelet transform zero-crossing. In: IEEE International Carnahan Conference on Security Technology, pp. $272-277$ (2001)

23. Donida Labati, R., Piuri, V., Scotti, F.: Agent-based image iris segmentation and multiple views boundary refining. In: Proceedings of the 3rd IEEE international conference on Biometrics: Theory, applications and systems, (BTAS'09), pp. 204 - 210. IEEE Press, Piscataway, NJ, USA (2009)

24. Donida Labati, R., Piuri, V., Scotti, F.: Neural-based iterative approach for iris detection in iris recognition systems. IEEE Symposium on Computational Intelligence for Security and Defence Applications pp. 1 - 6 (2009). 978-1-4244-3763-4

25. Donida Labati, R., Scotti, F.: Noisy iris segmentation with boundary regularization and reflections removal. Image Vision Comput. 28(2), 270 - 277 (2010). 0262-8856

26. Du, Y., Arslanturk, E., Zhou, Z., Belcher, C.: Video-based non-cooperative iris image segmentation. IEEE Trans. Syst., Man, Cybern. B, Cybern. 41(1), 64 - 74 (2011)

27. Feng, X., Fang, C., Ding, X., Wu, Y.: Iris localization with dual coarse-to-fine strategy. In: 18th International Conference on Pattern Recognition (ICPR 2006), vol. 4, pp. 553 - 556 (2006)

28. Fierrez, J., Ortega-Garcia, J., Toledano, D.T., Gonzalez-Rodriguez, J.: BioSec baseline corpus: A multimodal biometric database. Pattern Recogn. 40(4), 1389 - 1392 (2007)

29. Fitzgibbon, A., Pilu, M., Fisher, R.: Direct least square fitting of ellipses. IEEE Trans. Pattern Anal. Mach. Intell. 21(5), 476 - 480 (1999)

30. Gamassi, M., Lazzaroni, M., Misino, M., Piuri, V., Sana, D., Scotti, F.: Quality assessment of biometric systems: a comprehensive perspective based on accuracy and performance measurement. IEEE Trans. Instrum. Meas. 54(4), 1489 - 1496 (2005)

31. Gonzalez, R.C., Woods, R.E.: Digital Image Processing (3rd Edition). Prentice-Hall, Inc., Upper Saddle River, NJ, USA (2006)

32. He, Z., Tan, T., Sun, Z.: Iris localization via pulling and pushing. In: 18th International Conference on Pattern Recognition (ICPR 2006), vol. 4, pp. 366 - 369 (2006)

33. He, Z., Tan, T., Sun, Z., Qiu, X.: Robust eyelid, eyelash and shadow localization for iris recognition. In: 15th IEEE International Conference on Image Processing (ICIP 2008), pp. 265 $268(2008)$

34. He, Z., Tan, T., Sun, Z., Qiu, X.: Toward accurate and fast iris segmentation for iris biometrics. IEEE Trans. Pattern Anal. Mach. Intell. 31, 1670 - 1684 (2009)

35. Huang, S.C., Wu, Y.L., Hung, W.C., Tang, C.Y.: Point-of-regard measurement via iris contour with one eye from single image. In: IEEE International Symposium on Multimedia (ISM), pp. $336-341(2010)$

36. Institute of Automation, Chinese Academy of Sciences: CASIA Iris Database. http://biometrics.idealtest.org/ 
37. Ives, R., Kennell, L., Gaunt, R., Etter, D.: Iris segmentation for recognition using local statistics. In: Conference Record of the Thirty-Ninth Asilomar Conference on Signals, Systems and Computers, pp. 859 - 863 (2005)

38. Jain, A.K., Ross, A., Prabhakar, S.: An introduction to biometric recognition. IEEE Trans. Circuits Syst. Video Technol. 14, 4 - 20 (2004)

39. Jarjes, A., Wang, K., Mohammed, G.: Improved greedy snake model for detecting accurate pupil contour. In: 3rd Internatoional Conference on Advanced Computer Control (ICACC 2011), pp. 515 - 519 (2011)

40. Jeong, D.S., Hwang, J.W., Kang, B.J., Park, K.R., Won, C.S., Park, D.K., Kim, J.: A new iris segmentation method for non-ideal iris images. Image Vision Comput. 28, 254 - 260 (2010)

41. Jin, L., Xiao, F., Haopeng, W.: Iris image segmentation based on k-means cluster. In: 2010 IEEE International Conference on Intelligent Computing and Intelligent Systems (ICIS), vol. 3, pp. $194-198$ (2010)

42. Kennell, L., Ives, R., Gaunt, R.: Binary morphology and local statistics applied to iris segmentation for recognition. In: IEEE International Conference on Image Processing (ICIP 2006), pp. $293-296(2006)$

43. Li, P., Liu, X., Xiao, L., Song, Q.: Robust and accurate iris segmentation in very noisy iris images. Image Vision Comput. 28, 246 - 253 (2010)

44. Li, Y.H., Savvides, M.: Iris recognition, overview. In: S.Z. Li, A.K. Jain (eds.) Encyclopedia of Biometrics, pp. 810 - 819 (2009)

45. Liu-Jimenez, J., Sanchez-Reillo, R., Fernandez-Saavedra, B.: Iris biometrics for embedded systems. IEEE Trans. Very Large Scale Integr. (VLSI) Syst. 19(2), 274 - 282 (2011)

46. Luengo-Oroz, M.A., Faure, E., Angulo, J.: Robust iris segmentation on uncalibrated noisy images using mathematical morphology. Image Vision Comput. 28, 278 - 284 (2010)

47. Ma, L., Tan, T., Wang, Y., Zhang, D.: Efficient iris recognition by characterizing key local variations. IEEE Trans. Image Process. 13, 739 - 750 (2004)

48. Masek, L., Kovesi, P.: Matlab source code for a biometric identification system based on iris patterns (2003)

49. Phillips, P., Bowyer, K., Flynn, P., Liu, X., Scruggs, W.: The iris challenge evaluation 2005. In: IEEE International Conference on Biometrics: Theory, Applications and Systems, pp. 1 $8(2008)$

50. Phillips, P., Flynn, P., Scruggs, T., Bowyer, K., Chang, J., Hoffman, K., Marques, J., Min, J., Worek, W.: Overview of the face recognition grand challenge. In: IEEE Computer Society Conference on Computer Vision and Pattern Recognition, vol. 1, pp. 947 - 954 (2005)

51. Phillips, P., Moon, H., Rauss, P., Rizvi, S.: The feret evaluation methodology for facerecognition algorithms. In: IEEE Computer Society Conference on Computer Vision and Pattern Recognition, pp. 137 - 143 (1997)

52. Phillips, P., Scruggs, W., O’Toole, A., Flynn, P., Bowyer, K., Schott, C., Sharpe, M.: FRVT 2006 and ICE 2006 Large-Scale Experimental Results. IEEE Trans. Pattern Anal. Mach. Intell. 32(5), 831 - 846 (2010)

53. Proença, H.: Iris recognition: On the segmentation of degraded images acquired in the visible wavelength. IEEE Trans. Pattern Anal. Mach. Intell. 32(8), 1502 - 1516 (2010)

54. Proença, H., Alexandre, L.: UBIRIS: A noisy iris image database. In: 13th International Conference on Image Analysis and Processing, vol. LNCS 3617, pp. 970-977. Springer (2005)

55. Proença, H., Alexandre, L.: Iris segmentation methodology for non-cooperative recognition. IEEE Proceedings on Vision, Image and Signal Processing 153(2), 199 - 205 (2006)

56. Proença, H., Alexandre, L.: The NICE.I: Noisy Iris Challenge Evaluation - Part I. In: First IEEE International Conference on Biometrics: Theory, Applications, and Systems, pp. 1 - 4 (2007)

57. Proença, H., Filipe, S., Santos, R., Oliveira, J., Alexandre, L.: The UBIRIS.v2: A Database of Visible Wavelength Iris Images Captured On-the-Move and At-a-Distance. IEEE Trans. Pattern Anal. Mach. Intell. 32(8), 1529 - 1535 (2010)

58. Puhan, N., Sudha, N., Jiang, X.: Robust eyeball segmentation in noisy iris images using Fourier spectral density. In: 6th International Conference on Information, Communications and Signal Processing, pp. 1 - 5 (2007) 
59. Pundlik, S., Woodard, D., Birchfield, S.: Non-ideal iris segmentation using graph cuts. In: IEEE Computer Society Conference on Computer Vision and Pattern Recognition Workshops, pp. $1-6$ (2008)

60. Ren, X., Peng, Z., Zeng, Q., Peng, C., Zhang, J., Wu, S., Zeng, Y.: An improved method for Daugman's iris localization algorithm. Comput. Biol. Med. 38(1), 111 - 115 (2008)

61. RNCOS (ed.): Electronics Security: Global Biometric Forecast to 2012 (2010)

62. Rongnian, T., Shaojie, W.: Improving iris segmentation performance via borders recognition. In: 2011 International Conference on Intelligent Computation Technology and Automation (ICICTA), vol. 2, pp. 580 - 583 (2011)

63. Ross, A., Shah, S.: Segmenting non-ideal irises using geodesic active contours. In: 2006 Biometrics Symposium: Special Session on Research at the Biometric Consortium Conference, pp. $1-6(2006)$

64. Ryan, W., Woodard, D., Duchowski, A., Birchfield, S.: Adapting starburst for elliptical iris segmentation. In: IEEE International Conference on Biometrics: Theory, Applications and Systems, pp. $1-7$ (2008)

65. Sankowski, W., Grabowski, K., Napieralska, M., Zubert, M., Napieralski, A.: Reliable algorithm for iris segmentation in eye image. Image Vision Comput. 28, 231 - 237 (2010)

66. Schuckers, S., Lopez, P.M., Johnson, P., Sazonova, N., Hua, F., Lazarick, R., Miles, C., Talbassi, E., Sazonov, E., Ross, A., Hornak, L.: Quality-face / iris research ensemble (Q-FIRE) data collection steps, technical report. Clarkson University, Dept of Electrical and Computer Engineering (2010)

67. Schuckers, S., Lopez, P.M., Johnson, P., Sazonova, N., Hua, F., Lazarick, R., Miles, C., Talbassi, E., Sazonov, E., Ross, A., Hornak, L.: Quality-face / iris research ensemble (Q-FIRE) dataset overview, technical report. Clarkson University, Dept of Electrical and Computer Engineering (2010)

68. Shah, S., Ross, A.: Generating synthetic irises by feature agglomeration. In: IEEE International Conference on Image Processing, pp. 317 - 320 (2006)

69. Shah, S., Ross, A.: Iris segmentation using geodesic active contours. IEEE Trans. Inf. Forensics Security 4(4), $824-836$ (2009)

70. Shamsi, M., Saad, P., Ibrahim, S., Kenari, A.: Fast algorithm for iris localization using Daugman circular integro differential operator. In: International Conference of Soft Computing and Pattern Recognition, pp. 393 - 398 (2009)

71. Sreecholpech, C., Thainimit, S.: A robust model-based iris segmentation. In: International Symposium on Intelligent Signal Processing and Communication Systems (ISPACS 2009), pp. 599-602 (2009)

72. Sun, Z., Tan, T.: Ordinal measures for iris recognition. IEEE Trans. Pattern Anal. Mach. Intell. 31(12), 2211 - 2226 (2009)

73. Sundaram, R., Dhara, B., Chanda, B.: A fast method for iris localization. In: Second International Conference on Emerging Applications of Information Technology, pp. 89 - 92 (2011)

74. Tan, T., He, Z., Sun, Z.: Efficient and robust segmentation of noisy iris images for noncooperative iris recognition. Image Vision Comput. 28, 223 - 230 (2010)

75. Tian, Q.C., Pan, Q., Cheng, Y.M., Gao, Q.X.: Fast algorithm and application of hough transform in iris segmentation. In: International Conference on Machine Learning and Cybernetics, vol. 7, pp. 3977 - 3980 (2004)

76. Trucco, E., Razeto, M.: Robust iris location in close-up images of the eye. Pattern Analysis and Applications 8, 247 - 255 (2005)

77. Viola, P., Jones, M.: Rapid object detection using a boosted cascade of simple features. In: Proceedings of the 2001 IEEE Computer Society Conference on Computer Vision and Pattern Recognition (CVPR 2001), vol. 1, pp. 511 - 518 (2001)

78. West Virginia University: Biometric dataset collections. http://www.citer.wvu.edu/biometric_dataset_collections

79. Wildes, R.P.: Iris recognition: an emerging biometric technology. Proceedings of the IEEE 85(9), 1348 - 1363 (1997) 
80. Xu, G., Zhang, Z., Ma, Y.: Improving the performance of iris recogniton system using eyelids and eyelashes detection and iris image enhancement. In: 5th IEEE International Conference on Cognitive Informatics (ICCI 2006), vol. 2, pp. 871 - 876 (2006)

81. Yan, Y., Xie, M.: Eyelid and eyelash detection method based on morphology. In: 2nd International Conference on Computer and Automation Engineering (ICCAE 2010), vol. 1, pp. 678 $-681(2010)$

82. Zhang, X., Sun, Z., Tan, T.: Texture removal for adaptive level set based iris segmentation. In: 17th IEEE International Conference on Image Processing (ICIP 2010), pp. 1729 - 1732 (2010)

83. Zuo, J., Schmid, N.: On a methodology for robust segmentation of non ideal iris images. IEEE Trans. Syst., Man, Cybern. B, Cybern. 40(3), 703 - 718 (2010)

84. Zuo, J., Schmid, N.A., Chen, X.: On generation and analysis of synthetic iris images. IEEE Trans. Inf. Forensics Security 2(1), $77-90$ (2007) 